\title{
Bevacizumab as an adjuvant therapy for glioblastoma in elderly patients: the facts
}

\author{
Kristopher A. Lyon ${ }^{1,2}$, Jason H. Huang ${ }^{1,2}$ \\ ${ }^{1}$ Department of Neurosurgery, Baylor Scott \& White Health, Baylor Scott \& White Medical Center, Temple, TX, USA; ${ }^{2}$ Texas A\&M University \\ College of Medicine, Temple, TX, USA \\ Correspondence to: Jason H. Huang, MD, FACS. Baylor Scott \& White Health, Department of Neurosurgery, 2401 S. 31st Street, Temple, Texas \\ 76508, USA. Email: jason.huang@bswhealth.org. \\ Comment on: Wirsching HG, Tabatabai G, Roelcke U, et al. Bevacizumab plus hypofractionated radiotherapy versus radiotherapy alone in elderly \\ patients with glioblastoma: the randomized, open-label, phase II ARTE trial. Ann Oncol 2018;29:1423-30.
}

Submitted Jul 22, 2018. Accepted for publication Aug 10, 2018.

doi: $10.21037 /$ tcr.2018.08.19

View this article at: http://dx.doi.org/10.21037/tcr.2018.08.19

Glioblastoma (GBM) is the most aggressive and common primary brain tumor in adults, recurring in an average of 6.9 months after initial diagnosis even with aggressive treatment $(1,2)$. After initial diagnosis, most patients survive an average of 14 months (3). Since 2005, the standard of care for GBM that has provided a clinically significant survival benefit with minimal toxicity from adjuvant treatments has consisted of surgical resection, followed by conventional radiation therapy (RT) with concomitant and adjuvant temozolomide (TMZ) chemotherapy (4). These treatment guidelines, known as the Stupp protocol, were originally based off a large, randomized controlled trial using data obtained in patients less than 70 years old $(4,5)$. According to the most recent Central Brain Tumor Report of the United States (CBTRUS), GBM is found to have the highest average age-adjusted incident rates in patients age 65 to 84 years old and the lowest 1-, 2-, and 5-year relative survival rates when compared to younger patients diagnosed with a GBM (6). Because of this, many clinical studies have been performed to determine if the guidelines set forth in the Stupp protocol should likewise be applied to elderly patients with an intracranial GBM. Additionally, recent clinical trials testing the popular adjuvant chemotherapy bevacizumab (BEV) in the elderly population will be analyzed.

Compared to the younger population groups, most elderly patients present with diminished functional reserves, have additional comorbidities, and exhibit a slower recovery after surgery (7). Therefore, debate has existed regarding the surgical strategy, if any, for the elderly population.
In a recent large-scale retrospective study based on the Surveillance, Epidemiology, and End Results (SEER) registry, gross total resection of GBM improved overall survival (OS), even in the elderly population when compared to subtotal resection or no resection at all (8). A careful preoperative evaluation must be carried out though as elderly patients with a higher Karnofsky Performance Status (KPS) score, tumors in non-eloquent areas, methylated O-6-methylguanine-DNA-methyltransferase (MGMT) promoter status, and without a compromised neurological status are more likely to benefit from aggressive surgical resection (9).

RT remains a mainstay of treatment in the elderly population undergoing gross total resection of GBM, although the dosing pattern for this population group is under debate. A standard 6-week course of RT in the elderly population is associated with substantial risks of morbidity and early discontinuation, thus leading to investigations into shorter courses of radiation (5). In a recent prospective randomized clinical trial, elderly patients receiving the conventional 6 weeks of RT were compared against elderly patients receiving RT over 3 weeks (10). No difference in survival was found between the standard and the 3-week course of RT. In a more recent randomized controlled trial, elderly patients undergoing a short 1-week course of RT showed no differences in OS, progression-free survival (PFS), nor quality of life when compared to patients receiving 3 weeks or more of RT for GBM (11).

In addition to maximal safe resection and RT, Perry et al. showed patients greater than 65 years old with methylated 
forms of the MGMT promoter also benefit from TMZ in combination with RT (OS 13.5 months) versus RT alone (OS 7.7 months) (12). Patients with GBM with unmethylated forms of the MGMT promoter also showed increased OS to TMZ/RT treatment (OS 10.0 months) $v s$. RT alone (OS 7.9 months). Although TMZ has proven effective and safe, many trials have been performed to determine if other chemotherapeutic agents may provide additional benefit to the elderly population, specifically the anti-angiogenic agent $\mathrm{BEV}$.

Since receiving Food and Drug Administration approval in 2009 for the use in patients with GMB, BEV has safely been used in combination with TMZ and other agents in the treatment of primary and recurrent GBM (13). As an immunotherapy agent, BEV acts by blocking the interaction of vascular endothelial growth factor A (VEGF-A), an important signal protein, with the VEGF and neuropilin receptors $(14,15)$. These receptors, when activated, lead to endothelial cell proliferation with subsequent rapid tumor growth. Although many high-quality, phase 3 randomized control trials have tested the addition of $\mathrm{BEV}$ to the treatment regimen for younger patients with GBM, the elderly population has not been well represented $(16,17)$.

In a retrospective study, BEV was shown to be more beneficial in certain groups of patients versus others, namely the elderly and patients with a lower KPS score. In a retrospective study, Nghiemphu et al. compared a group of 44 patients with recurrent GBM greater than 55 years old with a KPS less than 80 treated with BEV compared against a control group receiving no additional treatments (18). Results indicated an increased PFS by 2.4 months and OS by 2.9 months in the group treated with BEV. In this study cohort, patients aged greater than 55 years old showed a 1.4-fold higher expression of VEGF-A when compared to younger patients, possibly accounting for the observed increase in PFS and OS. Furthermore, dexamethasone requirements were reduced in patients on $\mathrm{BEV}$.

Results of this study spurred additional studies in the elderly. A retrospective study involving 120 patients was performed by Babu et al. in patients greater than 65 years old with a median KPS score of 80 (19). Patients were treated with maximum safe resection, followed by RT and TMZ. Approximately $60 \%$ of patients underwent treatment with additional agents, most commonly BEV or Irinotecan. Results indicated patients undergoing gross total resection who were of older age, with a higher baseline KPS score, and who were treated with BEV to have significantly increased survival when compared to patients undergoing subtotal tumor resection. Furthermore, patients those treated with $\mathrm{BEV}$ versus Irinotecan or no agents at all had a significant increase in OS.

A prospective study by Reyes-Botero et al. sought to evaluate the effects of $\mathrm{BEV}$ in combination with $\mathrm{TMZ}$ in a group of 66 patients greater than 70 years old with a KPS $<70$ as the initial treatment after diagnosis of GBM (20). Comparing this patient group treated with TMZ/ BEV combination therapy with a similarly aged group undergoing no surgical, radiation, or chemotherapies for a newly diagnosed GBM revealed an increase in the OS by 12 weeks. In fact, one third of patients became autonomous and capable of self-care (KPS > 70). Although TMZ/BEV combination therapy may sound promising in this population group, a study performed years prior using only TMZ in the same population group yielded similar improvement in OS with the same proportion of patients becoming autonomous and capable of self-care (21). Since TMZ is less effective in patients harboring a nonmethylated MGMT promoter, additional patients may benefit when BEV is combined with TMZ therapy $(20,21)$.

To truly determine if past retrospective and prospective studies performed with BEV benefitted the elderly population, Wirsching et al. performed the first randomized control study in a group of 75 elderly patients with GBM treated with RT combined with BEV to patients receiving hypofractionated RT only (22). Patients greater than 65 years old with a KPS of $>60$ were enrolled in this trial who had undergone surgical resection of GBM within the last 28 to 49 days. Patients selected for this trial had a non-methylated form of the MGMT promoter since previous studies have already shown elderly patients with a methylated form of MGMT are best treated with TMZ therapy with or without hypofractionated RT (5). Patients receiving $\mathrm{BEV}$ in combination with $\mathrm{RT}$ had a longer PFS by 2.8 months when compared to patients receiving hypofractionated RT only, but there was no significant difference in OS, with both groups tested having an OS of slightly over 12 months. Analyzing the results further indicated patients with the receptor tyrosine kinase I methylation subtype and proneural gene expression gene subtype had the strongest association of improved PFS from BEV treatment.

Although increased OS could not be supported with the first randomized controlled trial performed by Wirsching et al., PFS was seen to be improved in each clinical trial of elderly patients with GBM treated with BEV. In addition to PFS, BEV also has a notable steroid-sparing effect because 
of blood brain barrier and blood tumor barrier restoration that may help elderly patients wean off steroids sooner, thus improving quality of life (20). In addition, the amount of symptomatic radiation necrosis in the elderly patients may also be reduced with the BEV adjuvant therapy (23). Although PFS may be increased, the risk of adverse events associated with $\mathrm{BEV}$ needs to be considered, especially in the elderly population. In the randomized clinical trials above, patients on BEV experienced an increased amount of hypertension, proteinuria, pulmonary embolism, deep venous thrombosis, and wound healing complications $(16,17)$. Additional randomized clinical trials specifically investigating the elderly population with GBM are needed to guide practitioners to deliver the best pharmacological therapies while minimal risk to these patients.

\section{Acknowledgments}

Funding: The authors of this paper were supported in part by National Institutes of Health/NINDS R01 NS067435 (to JH Huang).

\section{Footnote}

Provenance and Peer Review: This article was commissioned and reviewed by the Section Editor Xian-Xin Qiu (Shanghai Proton and Heavy Ion Center (SPHIC), a.k.a. the Proton and Heavy Ion Center of Fudan University Shanghai Cancer Center (FUSCC), Shanghai, China).

Conflicts of Interest: Both authors have completed the ICMJE uniform disclosure form (available at http://dx.doi. org/10.21037/tcr.2018.08.19). The authors have no conflicts of interest to declare.

Ethical Statement: The authors are accountable for all aspects of the work in ensuring that questions related to the accuracy or integrity of any part of the work are appropriately investigated and resolved.

Open Access Statement: This is an Open Access article distributed in accordance with the Creative Commons Attribution-NonCommercial-NoDerivs 4.0 International License (CC BY-NC-ND 4.0), which permits the noncommercial replication and distribution of the article with the strict proviso that no changes or edits are made and the original work is properly cited (including links to both the formal publication through the relevant DOI and the license).
See: https://creativecommons.org/licenses/by-nc-nd/4.0/.

\section{References}

1. Liao KL, Huang S, Wu YP. The prognosis for patients with newly diagnosed glioblastoma receiving bevacizumab combination therapy: a meta-analysis. Onco Targets Ther 2018;11:3513-20.

2. Cetin B, Gonul II, Gumusay O, et al. Carbonic anhydrase IX is a prognostic biomarker in glioblastoma multiforme. Neuropathology 2018. [Epub ahead of print].

3. Stupp R, Hegi ME, Mason WP, et al. Effects of radiotherapy with concomitant and adjuvant temozolomide versus radiotherapy alone on survival in glioblastoma in a randomised phase III study: 5-year analysis of the EORTC-NCIC trial. Lancet Oncol 2009;10:459-66.

4. Stupp R, Mason WP, van den Bent MJ, et al. Radiotherapy plus concomitant and adjuvant temozolomide for glioblastoma. N Engl J Med 2005;352:987-96.

5. Malmström A, Grønberg BH, Marosi C, et al. Temozolomide versus standard 6-week radiotherapy versus hypofractionated radiotherapy in patients older than 60 years with glioblastoma: the Nordic randomised, phase 3 trial. Lancet Oncol 2012;13:916-26.

6. Ostrom QT, Gittleman H, Liao P, et al. CBTRUS Statistical Report: Primary brain and other central nervous system tumors diagnosed in the United States in 20102014. Neuro Oncol 2017;19:v1-v88.

7. Karsy M, Yoon N, Boettcher L, et al. Surgical treatment of glioblastoma in the elderly: the impact of complications. J Neurooncol 2018;138:123-32.

8. Noorbakhsh A, Tang JA, Marcus LP, et al. Grosstotal resection outcomes in an elderly population with glioblastoma: a SEER-based analysis. J Neurosurg 2014;120:31-9.

9. Pessina F, Navarria P, Cozzi L, et al. Is surgical resection useful in elderly newly diagnosed glioblastoma patients? Outcome evaluation and prognostic factors assessment. Acta Neurochir 2018;160:1779-87.

10. Roa W, Brasher PM, Bauman G, et al. Abbreviated course of radiation therapy in older patients with glioblastoma multiforme: a prospective randomized clinical trial. J Clin Oncol 2004;22:1583-8.

11. Roa W, Kepka L, Kumar N, et al. International atomic energy agency randomized phase III study of radiation therapy in elderly and/or frail patients with newly diagnosed glioblastoma multiforme. J Clin Oncol 2015;33:4145-50. 
12. Perry JR, Laperriere N, O'Callaghan CJ, et al. Shortcourse radiation plus temozolomide in elderly patients with glioblastoma. N Engl J Med 2017;376:1027-37.

13. Blumenthal DT, Mendel L, Bokstein F. The optimal regimen of bevacizumab for recurrent glioblastoma: does dose matter? J Neurooncol 2016;127:493-502.

14. Plate KH, Breier G, Weich HA, et al. Vascular endothelial growth factor is a potential tumor angiogenesis factor in human gliomas in vivo. Nature 1992;359:845-8.

15. Tamura R, Tanaka T, Miyake K, et al. Bevacizumab for malignant gliomas: current indications, mechanisms of action and resistance, and markers of response. Brain Tumor Pathol 2017;34:62-77.

16. Gilbert MR, Dignam JJ, Armstrong TS, et al. A randomized trial of bevacizumab for newly diagnosed glioblastoma. N Engl J Med 2014;370:699-708.

17. Chinot OL, Nishikawa R, Mason W, et al. Upfront bevacizumab may extend survival for glioblastoma patients who do not receive second-line therapy: an exploratory analysis of AVAglio. Neuro Oncol 2016;18:1313-8.

18. Nghiemphu PL, Liu W, Lee Y, et al. Bevacizumab and chemotherapy for recurrent glioblastoma. Neurology 2009;72:1217-22.

Cite this article as: Lyon KA, Huang JH. Bevacizumab as an adjuvant therapy for glioblastoma in elderly patients: the facts. Transl Cancer Res 2018;7(Suppl 7):S802-S805. doi: 10.21037/ tcr.2018.08.19
19. Babu R, Komisarow JM, Agarwal VJ et al. Glioblastoma in the elderly: the effect of aggressive and modern therapies on survival. J Neurosurg 2016;124:998-1007.

20. Reyes-Botero G, Cartalat-Carel S, Chinot OL, et al. Temozolomide plus bevacizumab in elderly patients with newly diagnosed glioblastoma and poor performance status: an ANOCEF phase II trial (ATAG). Oncologist 2018;23:524-e44.

21. Gállego Pérez-Larraya J, Ducray F, Chinot O, et al. Temozolomide in elderly patients with newly diagnosed glioblastoma and poor performance status: an ANOCEF phase 2 trial. J Clin Oncol 2011;29:3050-5.

22. Wirsching HG, Tabatabai G, Roelcke U, et al. Bevacizumab plus hypofractionated radiotherapy versus radiotherapy alone in elderly patients with glioblastoma: the randomized, open-label, phase II ARTE trial. Ann Oncol 2018;29:1423-30.

23. Matsuda KI, Sakurada K, Nemoto K, et al. Treatment outcomes of hypofractionated radiotherapy combined with temozolomide followed by bevacizumab salvage therapy in glioblastoma patients aged $>75$ years. Int J Clin Oncol 2018. [Epub ahead of print]. 